\title{
Port-Access Thoracoscopic Anatomical Segmentectomy for Pediatric Intralobar Pulmonary Sequestration
}

\author{
Takashi Inoue ${ }^{1,2}$ Hiroyuki Oizumi ${ }^{2}$ Megumi Nakamura ${ }^{2}$ Mitsuaki Sadahiro $^{2}$ \\ ${ }^{1}$ Department of General Thoracic Surgery, Dokkyo Medical University, \\ Mibu, Tochigi, Japan \\ 2 Department of Surgery 2, Yamagata University, Yamagata, Japan \\ Address for correspondence Takashi Inoue, MD, Department of \\ General Thoracic Surgery, Dokkyo Medical University, 880 \\ Kitakobayashi, Mibu, Tochigi 3210293, Japan \\ (e-mail: takashi_inoue1980@yahoo.co.jp).
}

Thorac Cardiovasc Surg Rep 2014;3:42-44.
Abstract
Keywords
- thoracic surgery
- minimally invasive surgery
- lung
- congenital disorder
- pediatric
- thoracoscopy/VATS
- computed tomography

This report describes successful port-access thoracoscopic anatomical left lateral and posterior basal $(S 9+10)$ lung segmentectomy performed for intralobar pulmonary sequestration (ILPS) in a 5-year-old girl with recurrent pneumonia. Computed tomography revealed a multilocular lung abscess and an anomalous artery arising from the left gastric artery supplying the affected segment. After diagnosing ILPS, we performed thoracoscopic anatomical $\mathbf{S 9}+10$ segmentectomy. We consider thoracoscopic lung segmentectomy to be an important therapeutic option for pediatric ILPS.

\section{Introduction}

Intralobar pulmonary sequestration (ILPS)-an uncommon congenital malformation-comprises a nonfunctional segment contained within normal pulmonary parenchyma with an aberrant systemic artery vascularizing the segment. The definitive treatment is surgical excision, with the conventional approach consisting of the division of the anomalous artery through standard thoracotomy followed by lobectomy. We present here a case wherein port-access thoracoscopic anatomical left lateral and posterior basal (S9 + 10) lung segmentectomy was successfully performed in a pediatric patient with ILPS.

\section{Case Presentation}

A 5-year-old girl presented with high fever and cough at a local hospital. The patient had been hospitalized with pneumonia 1 year before the present admission. She was again diagnosed with pneumonia and treated with antibiotic ther- apy. As computed tomography (CT) revealed multilocular lung abscesses in the left lower lung ( $\mathbf{- F i g . ~ 1 A ) , ~ s h e ~ w a s ~}$ referred to our hospital.

Repeat CT revealed a multilocular lung abscess in the left lower lung and the presence of an anomalous artery arising from the left gastric artery, supplying the sequestration lung segment through the pulmonary ligament (-Fig. 1B). CT imaging also revealed deficient pulmonary artery perfusion and impaired bronchial perfusion into the left posterior basal lung. Therefore, the patient was diagnosed with ILPS, and thoracoscopic left $\mathrm{S} 9+10$ segmentectomy was planned.

Under general anesthesia with single-lung ventilation achieved by occluding the left bronchus using a Fogarty balloon catheter, the patient was placed in the right decubitus position. We used an image overlay of three-dimensional CT (3D-CT) on her body surface to determine the port sites (-Fig. 1C). The surgeon was positioned on the anterior side of the patient. A total of four incisions were made for the ports: a 15-mm flexible port on the anterior axillary line in the fifth intercostal space, a 5-mm port on the middle axillary line in
License terms

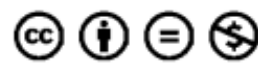

received

February 13, 2014

accepted

March 31, 2014

published online

June 9, 2014
DOI http://dx.doi.org/

10.1055/s-0034-1377065. ISSN 2194-7635. (c) 2014 Georg Thieme Verlag KG Stuttgart · New York 


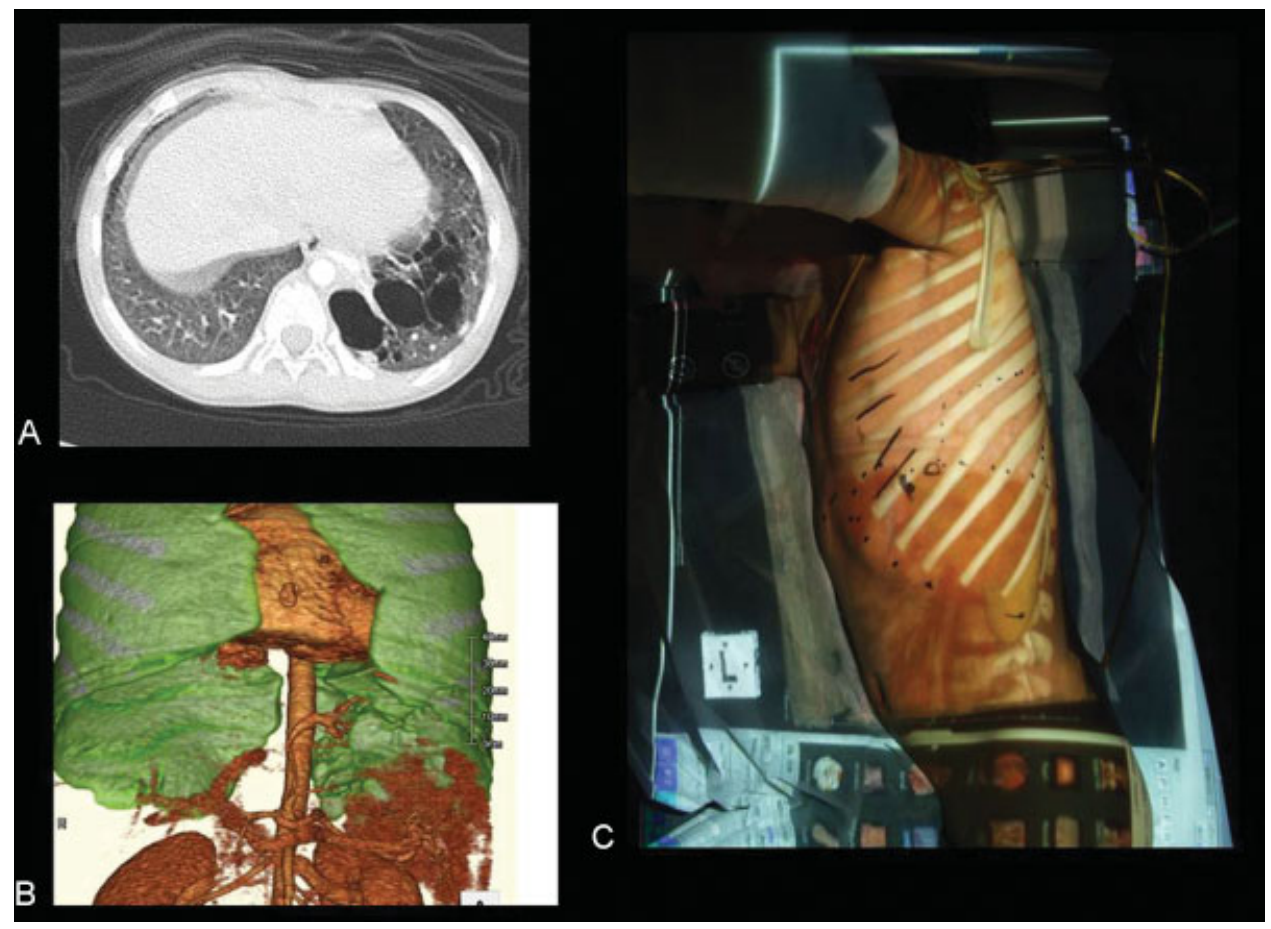

Fig. 1 Computed tomography (CT) revealed a multilocular lung abscess in the left lower lung (A). Three-dimensional CT (3D-CT) revealed the presence of an anomalous artery arising from the left gastric artery, supplying the sequestration lung segment through the pulmonary ligament (B). We used an image overlay of 3D-CT on the patient's body surface to determine the port sites (C).

the seventh intercostal space, and two 3-mm ports on the posterior axillary line in the fourth and seventh intercostal spaces. A 30-degree 5-mm scope was used. Thoracoscopic images showed an incompletely deflated segment with super- ficial parenchyma occupying one-third of the whole left lower lobe. We identified the anomalous artery in the pulmonary ligament and divided it using vessel clips ( - Fig. 2A, B). The lateral basal artery (A9) was isolated in the intralobar space

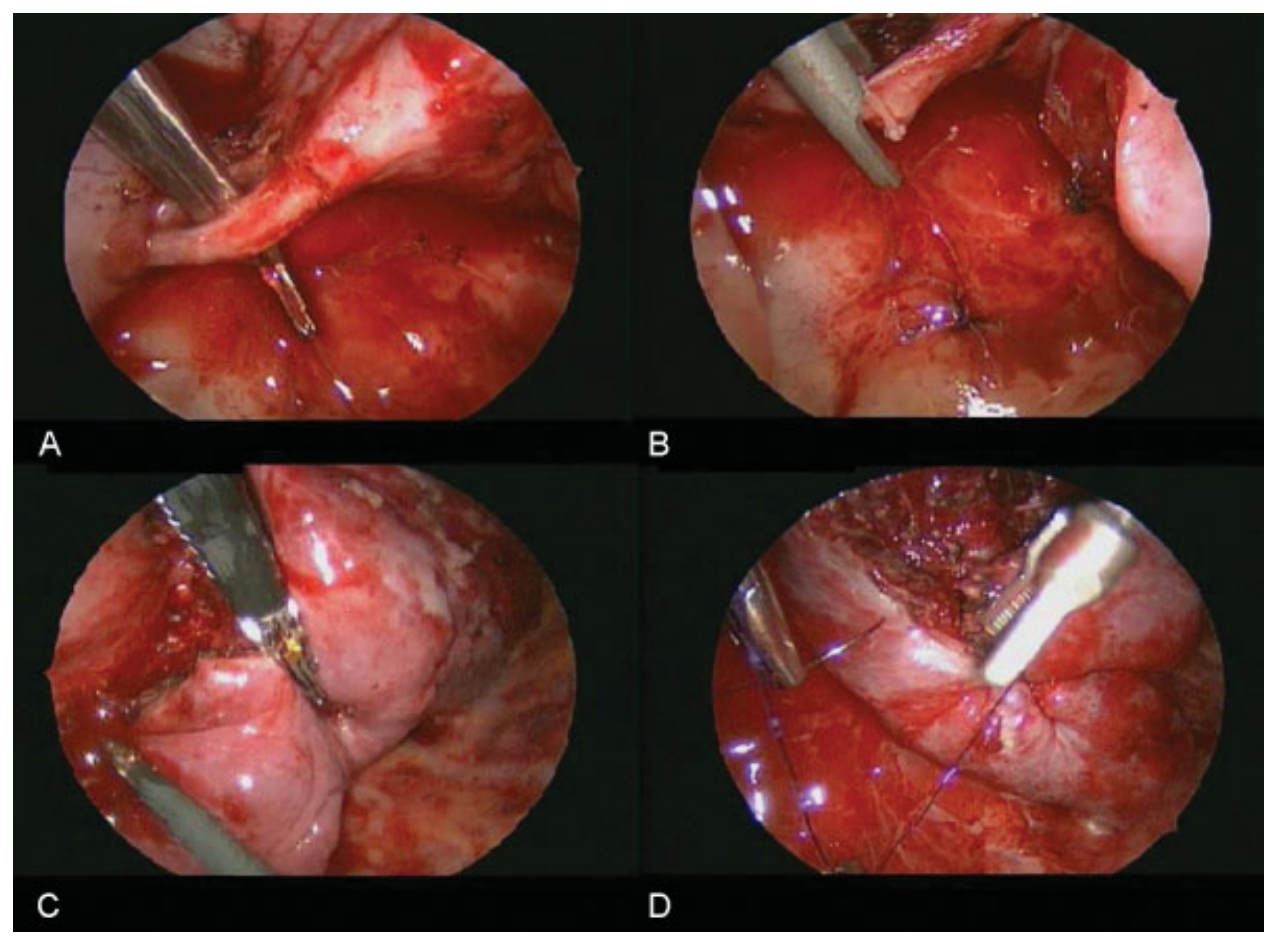

Fig. 2 We identified the anomalous artery in the pulmonary ligament (A) and divided it using vessel clips (B). S9 and S10 were incised along the demarcation line of the sequestration by means of a vessel sealing system (C). The remaining parenchymal surface of the S8 and S6 segments were closed with running sutures using a soluble monofilament (D). 
and was clipped and divided using an ultrasonically activated device. Then, we dissected the inferior basal vein and divided it using vessel clips. The segments 9 and $10($ S9 +10$)$ were incised along the demarcation line of the sequestration by means of a vessel sealing system (-Fig. $\mathbf{2 C}$ ). The border between $\mathrm{S} 9+10$ and the normal lung was identified by dissecting the intersegmental veins and based on the inflation-deflation line that was spontaneously created by the incomplete deflation and emphysematous changes in the ILPS legion. We also incised a portion of the parenchyma on the normal side of the lung adjacent to the inflation-deflation line to avoid postoperative infection of the preserved lung. The lateral basal bronchus (B9) was identified during the dissection of the parenchymal incision of the $S 9+10$ segments, and B9 was then divided using vessel clips. Subsequently, we ligated A9 and B9 simultaneously using thoracoscopic ligature devices. The remaining parenchymal surfaces of the S8 and S6 segments were closed with running sutures using a soluble monofilament (-Fig. 2D).

The total operation time was 288 minutes, and total blood loss was $127 \mathrm{~mL}$. The postoperative course was uneventful, and the chest drainage tube was removed on postoperative day 1 . The patient was discharged from the hospital on postoperative day 6 without any complications. CT imaging performed at 5 months postoperatively revealed good expansion of the preserved lung $(\mathrm{S} 6+8)$; the patient has remained healthy.

\section{Discussion}

Pulmonary sequestration is a congenital disorder characterized by the presence of an abnormal lung segment that lacks communication with the normal tracheobronchial tree and is vascularized by an aberrant systemic artery. The blood supply is commonly derived from the descending thoracic aorta and less frequently from the upper abdominal aorta and celiac artery. Pulmonary sequestration has been classified into two types: the intralobar type, wherein the segment is located in the normal pulmonary parenchyma without its own pleural covering, and the extralobar type, which is an isolated segment with its own pleural envelope. ${ }^{1}$ The venous drainage in ILPS is typically via the normal pulmonary venous system. ILPS is generally diagnosed during childhood, with most cases of ILPS presenting with recurrent episodes of bronchitis or pneumonia.

The primary treatment for ILPS is surgical resection. Conventional therapy comprised lobectomy through major thoracotomy to excise the affected lesion ${ }^{1}$; recently, the feasibility of video-assisted thoracic surgery (VATS) resection for ILPS has been demonstrated. ${ }^{2,3}$ VATS is associated with lower postoperative pain and faster recovery as compared with conventional surgery. Further, VATS has been shown to preserve early postoperative pulmonary function. ${ }^{4}$ Surgery for ILPS involves varying extents of resection, including wedge resection, segmentectomy, and lobectomy. ${ }^{1-3}$ Most patients with ILPS are treated early in life and, in the future, may often require major pulmonary resection for an acquired pulmonary disease, such as malignancy. A potential advantage of segmentectomy as compared with lobectomy is the preservation of pulmonary function. In our patient, we performed anatomical segmentectomy; therefore, the preserved lung ( $\mathrm{S} 6+8)$ had considerable volume, which may have enabled the preservation of pulmonary function.

Segmentectomy has been considered technically difficult in certain segments, particularly $\$ 9+10 .^{5}$ In such segments, digital dissection is necessary. In the present case, owing to the preoperative $3 \mathrm{D}-\mathrm{CT}$ simulation, we could determine the precise anatomy of the complex vessels and bronchi. Parenchyma-sparing resection is desirable in ILPS, unless the adjacent lung is involved in severe inflammatory processes; in such cases, preoperative 3D-CT simulation is highly useful in planning the operative strategy. Furthermore, in pediatric thoracoscopic surgery, which affords only limited working space, the vessel-sealing device was effective and helpful for exposing and dissecting pulmonary vessels and identifying the demarcation between the segments.

In ILPS, the aberrant artery arises directly from the systemic arterial tree. In our patient, it arose from the left gastric artery. It is therefore important to remember that accidental injury to the artery can lead to catastrophic hemorrhage. Therefore, preoperative delineation of the vasculature, for example, with $3 \mathrm{D}-\mathrm{CT}$, is crucial for minimizing injury to the aberrant artery.

\section{Conclusion}

We have herein described thoracoscopic anatomical lung segmentectomy for pediatric ILPS. We believe that minimally invasive thoracoscopic lung segmentectomy is an important therapeutic option for this clinical entity.

\section{Disclosure \\ No disclosures.}

\section{References}

1 Halkic N, Cuénoud PF, Corthésy ME, Ksontini R, Boumghar M. Pulmonary sequestration: a review of 26 cases. Eur J Cardiothorac Surg 1998;14(2):127-133

2 Kestenholz PB, Schneiter D, Hillinger S, Lardinois D, Weder W. Thoracoscopic treatment of pulmonary sequestration. Eur J Cardiothorac Surg 2006;29(5):815-818

3 Klena JW, Danek SJ, Bostwick TK, Romero M, Johnson JA. Videoassisted thoracoscopic resection for intralobar pulmonary sequestration: single modality treatment with video-assisted thoracic surgery. J Thorac Cardiovasc Surg 2003;126(3):857-859

4 Nakata M, Saeki H, Yokoyama N, Kurita A, Takiyama W, Takashima S. Pulmonary function after lobectomy: video-assisted thoracic surgery versus thoracotomy. Ann Thorac Surg 2000;70(3): 938-941

5 Oizumi H, Kanauchi N, Kato H, et al. Anatomic thoracoscopic pulmonary segmentectomy under 3-dimensional multidetector computed tomography simulation: a report of 52 consecutive cases. J Thorac Cardiovasc Surg 2011;141(3):678-682 